\title{
General anaesthesia or conscious sedation for painful procedures in childhood cancer: the family's perspective
}

\author{
C Crock, C Olsson, R Phillips, G Chalkiadis, S Sawyer, D Ashley, S Camilleri, J Carlin, \\ P Monagle
}

See end of article for authors' affiliations

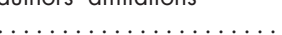

Correspondence to: Dr C Crock, Department of Laboratory Haematology, Royal Children's Hospital Parkville, 3052, Australia; catherine.crock@rch.org.au

Accepted 19 September 2002

\begin{abstract}
Background: Until recently, midazolam sedation was routinely used in our institution for bone marrow aspirates and lumbar punctures in children with cancer. It has been perceived by many doctors and nurses as being well tolerated by children and their families.

Aim: To compare the efficacy of inhalational general anaesthesia and midazolam sedation for these procedures.

Methods: A total of 96 children with neoplastic disorders, who received either inhalational general anaesthesia with sevoflurane, nitrous oxide, and oxygen (GA) or sedation with oral or nasal midazolam (SED) as part of their routine preparation for procedures were studied. The experiences of these childen were examined during their current procedure and during their first ever procedure. Main outcome measures were the degree of physical restraint used on the child, and the levels of distress and pain experienced by the child during the current procedure and during the first procedure. The family's preference for future procedures was also determined.

Results: During 102 procedures under GA, restraint was needed on four occasions $(4 \%)$ when the anaesthetic mask was first applied, minimal pain was reported, and children were reported as distressed about $25 \%$ of the time. During 80 SED procedures, restraint was required in $94 \%$, firm restraint was required in $66 \%$, the child could not be restrained in $14 \%$, median pain score was 6 (scale 0 (no pain) to 6 (maximum pain)), and $90 \%$ of the parents reported distress in their child. Ninety per cent of families wanted GA for future procedures. Many families reported dissatisfaction with the sedation regime and raised concerns about the restraint used on their child.

Conclusions: This general anaesthetic regime minimised the need for restraint and was associated with low levels of pain and distress. The sedation regime, by contrast, was much less effective. There was a significant disparity between the perceptions of health professionals and those of families with respect to how children coped with painful procedures.
\end{abstract}

M ultiple bone marrow aspirations and lumbar punctures are performed on children with leukaemia and other neoplastic disorders during the course of their illnesses. These procedures may give rise to considerable pain and distress in children. ${ }^{1-3}$ Anxiety can be extreme and often affects the children and their parents. ${ }^{12}$ Poor management of pain and distress may compromise treatment and place children at risk of depression and other long term psychological disorders. ${ }^{4-6}$

Regimes for the optimal management of pain and distress in children who require repeated procedures vary widely. ${ }^{7-9}$ Techniques used include sedation, ${ }^{10-12}$ general anaesthesia, ${ }^{513-15}$ and psychological therapies such as distraction, hypnotherapy, and relaxation techniques. ${ }^{31617}$ General anaesthesia is used more commonly in the United Kingdom ${ }^{7}$ and Sweden ${ }^{18}$ than in the United States. ${ }^{37}$ In more than half of Australian paediatric oncology units, sedation is used alone for the majority of these tests, using fixed doses of midazolam, doses titrated against response, or multiple drugs, in combinations such as midazolam and opioid. ${ }^{19}$ Literature comparing the use of sedation with general anaesthesia in paediatric oncology is sparse. We could find no data comparing families' opinions of these two pain management regimes in routine oncology practice.

In 1999, a general anaesthetic service was introduced at the Royal Children's Hospital, Melbourne, for children requiring bone marrow aspirations and lumbar punctures. Prior to this, sedation with a fixed dose of midazolam was routinely used for these procedures. The introduction of this general anaesthetic service has provided the opportunity to investigate the relative efficacies of these two regimes.

\section{METHODS}

Subjects

Between November 1999 and November 2000, children under 18 years of age attending the Royal Children's Hospital for bone marrow or lumbar puncture tests in the course of their treatment for neoplastic disorders were eligible to participate in this study, excluding those having their first investigative procedures prior to diagnosis. The decision to use either general anaesthesia (GA) or sedation (SED) was made by the child's clinic doctor based on the child's previous experiences and the doctor's assessment of the child's needs. This study was approved by the hospital's research ethics committee. Informed consent was obtained from the families who were not aware of any particular hypothesis relating to the study.

\section{General anaesthesia}

Procedures under GA were performed in the outpatient day surgery unit by a team, none of whom wore theatre clothes. The children were allowed to remain in their normal clothes and shoes. They were fasted from solids for five hours and from clear fluids for two hours prior to anaesthesia. Premedication was not used. Children were encouraged to select a

Abbreviations: GA, general anaesthesia; SED, sedation 
Table 1 Background characteristics based on type of pain management used at current procedure

\begin{tabular}{|c|c|c|c|}
\hline & $\mathrm{GA}(n=75)$ & $\operatorname{SED}(n=21)$ & Total $(n=96)$ \\
\hline $\begin{array}{l}\text { Gender } \\
\text { Female }\end{array}$ & 34 (45) & $8 \quad(38)$ & $42 \quad(44)$ \\
\hline \multicolumn{4}{|l|}{ Age (y) } \\
\hline $0-3$ & $16 \quad(21)$ & $6 \quad(29)$ & $22(23)$ \\
\hline $4-7$ & $21(28)$ & $8(38)$ & $29 \quad(30)$ \\
\hline $8-17$ & $38 \quad(51)$ & 7 (33) & $45 \quad(47)$ \\
\hline Mean & 7.8 & 6.4 & 7.5 \\
\hline \multicolumn{4}{|c|}{ Nature of current procedure } \\
\hline BMA only & $16 \quad(21)$ & 3 (14) & $19 \quad(20)$ \\
\hline LP only & $28 \quad(37)$ & $11 \quad(52)$ & 39 (41) \\
\hline$B M A+L P$ & 31 (41) & 7 (33) & $38 \quad(40)$ \\
\hline \multicolumn{4}{|c|}{ Number of previous procedures } \\
\hline $1-5$ & $21 \quad(28)$ & $7 \quad$ (33) & $28 \quad(29)$ \\
\hline $6-15$ & $34(45)$ & $11 \quad(52)$ & $45 \quad(47)$ \\
\hline $16-30$ & 20 (27) & 3 (14) & $23(24)$ \\
\hline \multicolumn{4}{|c|}{ Pain management used at first procedure } \\
\hline GA & $19 \quad(25)$ & $8 \quad(38)$ & $27 \quad(28)$ \\
\hline SED & $47 \quad(62)$ & $12 \quad(57)$ & 59 (61) \\
\hline Neither GA nor SED & 9 (12) & 1 (5) & $10 \quad(10)$ \\
\hline
\end{tabular}

Table 2 Degree of physical restraint required to administer the current procedure and the distress experienced by the child, as reported by parents

\begin{tabular}{|c|c|c|c|}
\hline & GA $(n=75)$ & $\operatorname{SED}(n=21)$ & $p$ value \\
\hline Restraint (n) & & & $<0.001$ * \\
\hline No restraint & 72 & 2 & \\
\hline Gentle restraint & 2 & 2 & \\
\hline Firm restraint & 1 & 2 & \\
\hline Very firm restraint & 0 & 13 & \\
\hline Unable to be held & 0 & 2 & \\
\hline \multicolumn{4}{|l|}{ Distress (n, \%) } \\
\hline Before procedure & 13 (17) & 7 (33) & 0.110 \\
\hline During procedure & 1 (1) & $18(86)$ & $<0.001$ \\
\hline After procedure & 7 (9) & $7(33)$ & 0.006 \\
\hline
\end{tabular}

scent (for example, chocolate) which was applied to the face mask before sevoflurane in 30\% nitrous oxide and oxygen were administered. Neither laryngeal masks nor endotracheal tubes were used. Routine monitoring was performed during the anaesthesia. Parents were present in the anaesthetic room during the induction, and later in the recovery room. Children were usually discharged home within 15-30 minutes of completion of the procedures.

\section{Sedation}

Procedures under SED were performed in a room in the outpatient clinic. These children were not required to fast prior to the procedures and their vital signs were not monitored. Local anaesthetic cream, AnGel (Royal Children's Hospital formulation, $4 \%$ amethocaine) was applied under occlusive dressings at the required sites 45 minutes before the intervention. Oral midazolam $0.4-0.6 \mathrm{mg} / \mathrm{kg}$ was given about $30 \mathrm{~min}-$ utes before the procedure; alternatively intranasal drops of midazolam $0.2-0.4 \mathrm{mg} / \mathrm{kg}$ were used about 10 minutes beforehand. Lignocaine (Lidocaine) $1 \%(2-5 \mathrm{ml}$ ) was injected subcutaneously and subperiosteally just prior to bone marrow procedures. In addition to the doctor who was performing the procedures, a nurse and parents were present. Children were discharged usually within 20-60 minutes of completion of the procedure.

\section{Questionnaire design}

Questionnaires comprising fixed format and free text questions were given to the children and to their parents immediately prior to the procedure, and in most cases were completed before the child went home. The questionnaires asked about the child's experiences just before, during, and just after the current procedure and also asked about the first procedure performed at the time of diagnosis on that child. The child/adolescent questionnaire was designed to investigate how scared or upset the child was about having the test, how much the test hurt, and the child's preference for future procedures. A simplified version was given to children between the ages of 4 and 7 . The parental questionnaire was designed to determine the degree of physical restraint needed to hold the child during the procedure, the degree of distress and pain that their child had experienced, and the parents' preference for future procedures.

Restraint and distress were assessed on Likert scales scored from "not held" to "unable to be held" and from "not scared/ not upset" to "extremely scared/extremely upset" respectively. 
Table 3 Background characteristics, based on type of pain management used at first procedure

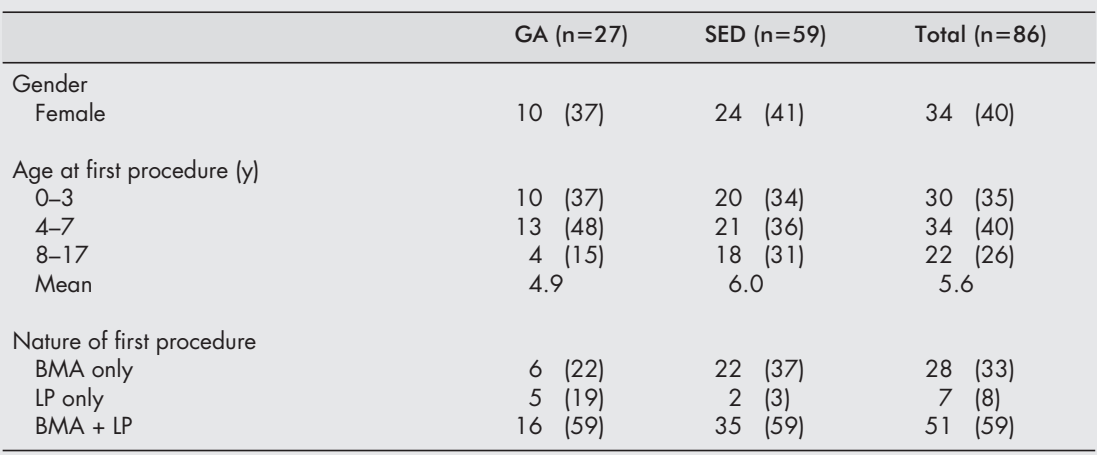

Results expressed as $n(\%)$.

BMA, bone marrow aspirate; LP, lumbar puncture; GA, general anaesthesia; SED, sedation.

Table 4 Degree of physical restraint required to administer the first procedure and the distress experienced by the child, as reported by parents

\begin{tabular}{lccr}
\hline & GA $(n=27)$ & SED $(n=59)$ & $p$ value \\
\hline Restraint $(n)$ & & 3 & $<0.001^{*}$ \\
No restraint & 26 & 5 & \\
Gentle restraint & 0 & 2 & \\
Firm restraint & 1 & 40 & \\
Very firm restraint & 0 & 9 & \\
Unable to be held & 0 & & \\
Distress (n, \%) & & $43(73)$ & $<0.206$ \\
Before procedure & $16(59)$ & $57(97)$ & $<0.001$ \\
During procedure & 0 & $43(73)$ & \\
After procedure & $7(26)$ & & \\
\hline GA, general anaesthesia; SED, sedation. & & \\
* $p<0.001$ for comparison between GA and SED groups of distribution of restraint. &
\end{tabular}

Children were regarded as distressed if they were reported as being very scared, extremely scared, very upset, or extremely upset. Pain was assessed using the Bieri Faces Pain Scale ${ }^{20}$ scored from 0 (no pain) to 6 (maximum pain). Space was provided on the form for parents and children to make general comments.

\section{Statistical analysis}

The frequency of various responses was compared between the sedated and anaesthetised episodes using Fisher's exact test. Pain scores were compared using the non-parametric Wilcoxon rank sum test.

\section{RESULTS}

From a cohort of 104 families, 96 families entered the study. The mean age of the children at enrolment was 7.5 years (range 1-17 years; 54 boys, 42 girls; table 1). The primary diagnoses were leukaemia $(n=83)$, brain tumour $(n=4)$, lymphoma $(n=2)$, neuroblastoma $(n=2)$, or other conditions $(n=5)$. All of these children had undergone lumbar puncture or bone marrow aspiration on at least one previous occasion, while half had experienced more than 10 previous procedures. During the current procedure, a child may have had either a bone marrow aspiration or a lumbar puncture, or both (table 1). Background characteristics were similar between the groups given GA and SED (table 1).

\section{Physical restraint (current procedure)}

In the GA group, three (4\%) children were restrained to enable the face mask to be applied. In the SED group, 19 (90\%) needed to be restrained. Of these, $13(68 \%)$ had to be held very firmly, and two (10\%) could not be restrained due to their level of distress (table 2).

\section{Distress (current procedure)}

Parents' reports of their child's distress before, during, or after the procedure showed considerably lower levels of distress in the GA group (table 2). From the self reports of children aged 4-17, the proportion in the GA group who reported they were distressed at some stage ( 6 of $59,10 \%$ ) was substantially lower than the proportion in the SED group (8 of 15, 53\%) $(\mathrm{p}=0.001)$.

\section{Pain (current procedure)}

Parents reported that the children who received GA experienced minimal pain (median pain score $=0$ ), whereas those who received SED experienced considerable pain (median pain score $=6)(\mathrm{p}<0.001)$. Reports from the children were consistent with this (GA median pain score $=0$, SED median pain score $=6, p<0.001)$.

\section{The child's first procedure}

Data about the first procedure on each child (performed at the time of diagnosis) were also collected in the questionnaires and were analysed separately. Data on the first procedures of 10 children who received no medication for their first procedure are not included. Of the 96 children in the study, 27 received GA on their first occasion and 59 received SED (table 3). Background characteristics were similar between the groups given GA and SED for their first procedure (table 3). Comparison of these two groups with respect to restraint (table 4), distress (table 4), and pain (parent reported median 
pain scores 0 (GA) and 6 (SED), p < 0.001) shows higher levels of distress in all groups, but otherwise similar findings to the analysis of the current procedures.

\section{Preferences for future procedures}

When asked about their preference for future bone marrow aspirations or lumbar punctures, $90 \%$ of $4-7$ year olds, and $91 \%$ of $8-17$ year olds preferred their next procedure to be performed under GA. Consistent with the children's choices, $90 \%$ of parents expressed a preference for the use of GA, $8 \%$ preferred the use of SED, and the remaining $2 \%$ (two parents) preferred that the procedures be done without anaesthesia or sedation.

\section{Qualitative comments}

Thirty eight families (40\%) in our study reported problems for their child that the families attributed to previous painful procedures under sedation. These included anxiety, prolonged screaming, attempts to run away, nausea, poor sleep, lack of enjoyment of other activities for days before any hospital visit, nightmares, abnormal behaviour, and marital problems. Twenty nine families (30\%) raised the issue of the physical restraint used on their child during procedures under sedation.

\section{DISCUSSION}

Pain management for children having recurrent procedures is an important paediatric problem. Successful pain management is manifested by the child who is not distressed by procedures, not merely by a child who can be held still. The results of our study suggest that the sedation regime used in our hospital does not meet this goal. The study revealed high levels of restraint, pain, distress, and parental concerns associated with SED. By contrast, GA was associated with minimal levels of restraint and pain, low levels of distress, and few parental concerns. There was a striking preference for future procedures to be done under GA.

It is of major significance that prior to this study, the children and their families were generally perceived by senior medical and nursing staff to be happy with the sedation regime and coping well. This view is in contrast to the patient and parental views in the study findings. Some parents commented that they had not previously expressed concerns because they had been unaware of any safe alternative to sedation. Parents and children may also be reluctant to express dissatisfaction with medical care to their oncologist. In particular, both parents and health professionals may find it difficult to examine the use of physical restraint in carrying out procedures on children. ${ }^{21}$ Prior to this study, we were not aware of any parent having complained about restraint being used on their child, yet 30\% of families raised concerns about this in their replies. Restraint in children requires further investigation.

The study was designed to assess the families' response to routine treatment and therefore was not randomised. This introduces a potential source of bias. However, a randomised trial would exclude all families with clear preferences for a particular pain management regime and would not address the question of how the families perceive their routine management. Jay et al emphasised that data about a pain management regime obtained from a randomised trial cannot necessarily be generalised to a clinical setting. ${ }^{3}$ In our study, $92 \%$ of eligible families completed the study, and virtually all children in the study (92 of 96) had experienced both GA and SED at some stage during their treatment. Moreover, the large clinical differences in the outcome measures between GA and SED (whether at the current or first procedure) cannot be attributed to the small differences in baseline characteristics shown in tables 1 and 3.
Decisions about whether a child would receive GA or SED at any particular procedure were made by the clinic doctor. It can be seen from tables 1 and 3 that more children had SED for their initial procedures, but many changed to GA. The most common reason to change was that a previous procedure under SED had been associated with notable distress or an inability to hold the child still. Children who had less distress with SED procedures were more likely to continue with SED. As such, patients who found procedures difficult are overrepresented in the GA group for the current procedure, further strengthening the study findings.

A possible confounder with this study is whether lumbar puncture data should be included with bone marrow aspirate data. Our results suggest combining data from these two procedures is reasonable. An analysis restricted to the 21 current SED episodes showed no significant statistical or clinical difference when the 11 children who only had a lumbar puncture were compared with the 10 children who had a bone marrow aspirate (with or without a lumbar puncture). Contrary to what might be expected, it is interesting that there was no increased tendency to favour GA for younger children, and the mean ages of our GA and SED groups are similar. This accords with observations of Jay and Katz who commented that older children may be highly anxious about procedures without exhibiting this in as obvious a manner as younger children. $^{12}$

We used a fixed dose of midazolam in this study. Many centres achieve more effective sedation by giving higher midazolam doses, titrating the dose intravenously against pain responses or adding an opioid analgesic agent. However, all of these measures require levels of patient preparation, monitoring, and staffing appropriate for anaesthesia. ${ }^{22}$ If titrated, or in the presence of opioids, sedation can unpredictably deepen or become general anaesthesia. Under such conditions, the assumption that monitoring, patient selection, personnel, and equipment can be chosen by the anticipated level of sedation is "tragically flawed". ${ }^{23}$ Indeed, Yaster and Maxwell have argued that a state of "conscious sedation" in which a child is simultaneously responsive to voice stimulus but immobile in the face of pain does not exist. ${ }^{23}$

Given our findings, it is worth considering what factors may prevent cancer units from offering general anaesthesia routinely to these children. Safety is often quoted. Many of the participating parents in this study had been told that the use of GA was less safe than SED. Given the known safety of short acting inhalational anaesthesia contrasted with reported adverse reactions and deaths in patients sedated with midazolam, ${ }^{10}{ }^{11}$ the opposite may be true. A second factor is the convenience of being able to do the procedure under sedation straight away. However, this convenience usually stems from not requiring the children to fast and giving them a lower level of monitoring and care, thereby increasing safety concerns. A third factor is cost, in particular the cost of an anaesthetist. However, in our institution, general anaesthesia offers several savings in costs that are not immediately evident. For example, the procedure is quicker, bone marrow specimens are more often of good quality resulting in more rapid and accurate diagnoses, procedures virtually never fail or need to be repeated, children may be less scared of any subsequent event in their treatment, and the risk of litigation from staff injured while restraining a child or from parents appears much less.

No single pain management regime is suitable for every child. Our study does not imply that all children require general anaesthesia. Some children may warrant general anaesthesia for procedures in the first period after diagnosis but then accept sedation when they feel secure in the hospital environment. Other children prefer sedation. In a few children, psychological and behavioural techniques are dramatically effective. A few require no sedation at all. What our study does show is the importance of actively seeking feedback from informed families about their child's responses to pain and to pain management. 


\section{Conclusion}

This study has shown that general anaesthesia administered through a face mask is well accepted by children having bone marrow aspirations or lumbar punctures. By contrast, when sedation alone was used, the children usually required considerable physical restraint and experienced unacceptable levels of pain and distress during the procedures. Our data suggest that there may be a significant disparity between the perceptions of health professionals and those of families with respect to how children are coping with painful procedures.

\section{Authors' affiliations}

C Crock, P Monagle, Department of Laboratory Haematology, Royal Children's Hospital, Melbourne, Australia

C Olsson, Murdoch Children's Research Institute, Royal Children's Hospital

R Phillips, S Sawyer, J Carlin, Department of Paediatrics, University of Melbourne, Parkville, Australia

G Chalkiadis, Department of Anaesthetics, Royal Children's Hospital D Ashley, S Camilleri, Department of Haematology, Royal Children's Hospital

\section{REFERENCES}

1 Katz ER, Kellerman J, Siegel SE. Behavioural distress in children with cancer undergoing medical procedures: developmental considerations. J Consult Clin Psychol 1980:48:356-65.

2 Jay $\mathrm{S}$, Ozolins $\mathrm{M}$, Elliott $\mathrm{CH}$. Assessment of children's distress during painful medical procedures. Health Psychol 1983;2:133-47.

3 Jay S, Fitzgibbon I, Woody P, et al. A comparative study of cognitive behaviour therapy versus general anaesthesia for painful medical procedures in children. Pain 1995:62:3-9.

4 Weisman S, Bernstein B, Schecter N. Consequences of inadequate analgesia during painful procedures in children. Arch Pediatr Adolesc Med 1998; 152:147-9.

5 Hain WR, Tomlinson JH, Barbor PR. Anaesthesia for minor procedures in children with malignant disease. J R Soc Med 1985;78:715-20.

6 Smalley A. Needle phobia. Paediatr Nurs 1999;11:17-20.

7 Hain RD, Campbell C. Invasive procedures carried out in conscious children: contrast between North American and European paediatric oncology centres. Arch Dis Child 2001;85:12-15.
8 Kazak AE, Penati $B$, Brophy $P$, et al. Pharmacologic and psychologic interventions for procedural pain. Pediatrics 1998;102(1 pt 1):59-66.

9 Kanagasundaram SLL, Cavalletto B, Keneally J, et al. Efficacy and safety of nitrous oxide in alleviating pain and anxiety during painful procedures. Arch Dis Child 2001;84:492-5.

10 Sievers TD, Yee JD, Foley ME, et al. Midazolam for conscious sedation during pediatric oncology procedures: safety and recovery parameters.

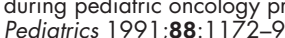

11 Bailey PPN, Ashburn M, Moll J, et al. Frequent hypoxemia and apnea after sedation with midazolam and fentanyl. Anaesthesiology 1990:73:826-30.

12 Sandler EWC, Conner K, Reilley K, et al. Midazolam versus fentanyl as premedication for painful procedures in children with cancer. Pediatrics 1992;89:631-4

13 Hertzog JH, Dalton HJ, Anderson BD, et al. Prospective evaluation of propofol anesthesia in the pediatric intensive care unit for elective oncology procedures in ambulatory and hospitalized children. Pediatrics 2000;106:742-7.

14 Fisher DM, Robinson S, Brett CM, et al. Comparison of enflurane, halothane, and isoflurane for diagnostic and therapeutic procedures in children with malignancies. Anesthesiology 1985;63:647-50.

15 Jayabose S, Levendoglu-Tugal O, Giaamelli J, et al. Intravenous anaesthesia with propofol for painful procedures in children with cancer J Pediatr Hematol Oncol 2001;23:290-3.

16 Jay SM, Elliott $\mathrm{CH}$, Ozolins $M$, et al. Behavioral management of children's distress during painful medical procedures. Behav Res Ther 1985;23:513-20.

17 Zeltzer L, LeBaron S. Hypnosis and nonhypnotic techniques for reduction of pain and anxiety during painful procedures in children and adolescents with cancer. J Pediatr 1982;101:1032-5.

18 Ljungman G, Gordh T, Sorensen S, et al. Lumbar puncture in pediatric oncology: conscious sedation vs. general anesthesia. Med Pediatr Oncol 2001;36:372-9.

19 Barnes C, Downie P, Chalkiadis G, et al. Sedation practices for Australian and New Zealand paediatric oncology patients. J Paediatr Child Health 2002;38:170-2.

20 Bieri D, Reeve RA, Champion GD, et al. The Faces Pain Scale for the self-assessment of the severity of pain experienced by children: development, initial validation, and preliminary investigation for ratio scale properties. Pain 1990;41:139-50.

21 Collins P. Restraining children for painful procedures. Paediatr Nurs $1999 ; 11: 14-16$

22 American Academy of Pediatrics Committee on Drugs. Guideline for monitoring and management of pediatric patients during and after sedation for diagnostic and therapeutic procedures. Pediatrics 1992;89:1 $110-15$

23 Maxwell L, Yaster $\dot{M}$. The myth of conscious sedation. Arch Pediatr Adolesc Med 1996:150:665-7. 